\title{
Impact loading of stepped circular plates
}

\author{
J. Lellep \& A. Mürk \\ Institute of Mathematics, University of Tartu, Estonia
}

\begin{abstract}
The inelastic response of stepped plates to concentrated loading is studied. An approximate analysis for determination of maximal residual deflections is developed for fully clamped plates. As an example, an eccentrically loaded circular plate is investigated in a greater detail.

Keywords: circular plate, inelastic material.
\end{abstract}

\section{Introduction}

In the literature one can find a number of exact theoretical predictions and numerical solutions of problems of dynamic plastic behaviour of inelastic beams, plates and shells (Jones [1], Kaliszky [2, 3]). Since exact theoretical solutions are available only for relatively simple cases (plastic bending of straight beams or plates and shells remaining axisymmetric during the dynamic deformation) it is reasonable to extend the list of theoretical predictions making use of various simplifications. In the present paper the dynamic plastic behaviour of circular plates subjected to eccentrically applied concentrated loadings is studied.

\section{Problem formulation and basic hypotheses}

Let us consider a thin walled plate with smooth boundary $r=r_{*}(\theta)$ presented in polar coordinates (fig. 1). It is assumed that the plate is loaded by a concentrated force $P=P(t)$ applied at the origin of coordinates.

In what follows, we consider the case of rectangular loading when

$$
P(t)=\left\{\begin{array}{l}
P_{0}, 0 \leq t \leq t_{0} \\
0, t>t_{0}
\end{array}\right.
$$

Here $t$ stands for the current time and $t_{0}$ is a fixed moment of time. As regards the intensity of the loading $P_{0}$ it is assumed to be of medium size according to 
the classification used in the rigid plastic analysis (see Jones [1], Lellep and Mürk [4]).

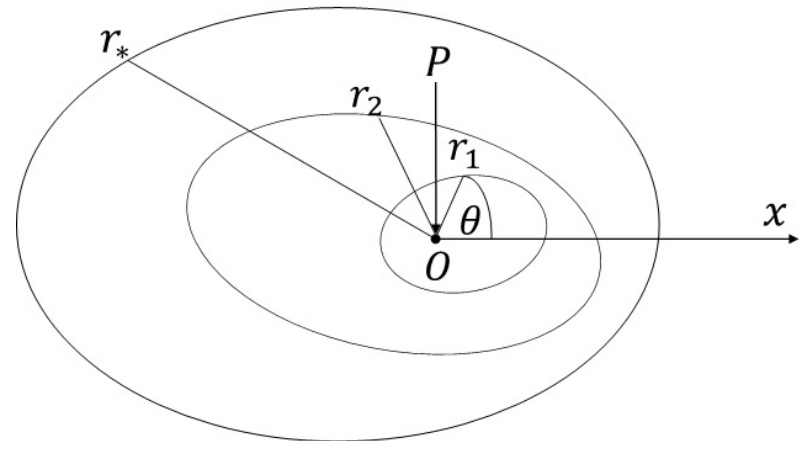

Figure 1: Stepped plate.

The material of the plate is assumed to be an ideal rigid-plastic material obeying the square yield conditions (Fig. 2).

Here $M_{1}, M_{2}$ stand for principal moments whereas $M_{00}=\sigma_{0} h_{0}^{2} / 4$ and $M_{01}=\sigma_{0} h_{1}^{2} / 4, \ldots, M_{0 n}=\sigma_{0} h_{n}^{2} / 4$, provided $h_{j}$ is the current thickness and $\sigma_{0}$ stands for the yield stress of the perfect plastic material. The thickness

$$
h=h_{j}
$$

for $r \in\left(r_{j}, r_{j+1}\right)$ where $j=0, \ldots, n$. Here $r_{j}=r_{j}(\theta)$ and $\theta \in(0,2 \pi)$. The contour of the plate is given by the equation

$$
r=r_{n+1}(\theta)=r_{*}(\theta)
$$

where $r_{n+1}(\theta)$ is a given differentiable function of the polar angle. It is assumed that the plate is fully clamped at the edge.

It is assumed that the displacement field can be determined according to the approximate method of mode form motions (Jones [1]). According to this concept the displacement rates can be presented in the form

$$
W(r, \theta, t)=W_{0}(t) f(r, \theta) .
$$

In eqn (4) the function $W_{0}(t)$ depends on time only. In the present paper the mode form corresponding to the conical deformation field

$$
f(r, \theta)=1-\frac{r}{r_{*}(\theta)}
$$

will be used. 


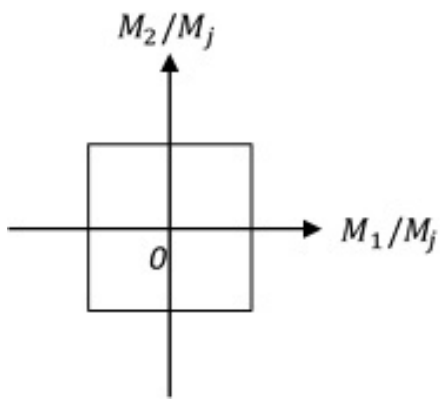

Figure 2: Yield condition.

The aim of the paper is to determine the approximate values of maximal residual deflections corresponding to the dynamic loading (1).

\section{External and internal energy dissipation}

Evidently, the external power caused by inertial forces can be calculated as

$$
\dot{D}_{e 1}=\iint_{S}-\mu \ddot{W} \dot{W} d S
$$

where $S$ stands for the surface of the middle plane of the plate and $\mu$ is the mass density. Here $\dot{W}$ is the deflection rate (velocity) and $\ddot{W}$ - the acceleration of points lying at the middle surface. The rate of the external work due to the concentrated loading is

$$
\dot{D}_{e 2}=P \dot{W}_{0}
$$

where according to eqns (4), (5)

$$
\dot{W}_{0}=\dot{W}(0, \theta, t) .
$$

Substituting (2), (4), (5) in (6) yields

$$
\dot{D}_{e 1}=-\mu \ddot{W}_{0} \dot{W}_{0} \int_{0}^{2 \pi} \sum_{j=0}^{n} \int_{r_{j}}^{r_{j+1}} h_{j}\left(1-\frac{r}{r_{*}}\right)^{2} r d r d \theta .
$$

The total external work rate is

$$
\dot{D}_{e}=\dot{D}_{e 1}+\dot{D}_{e 2}
$$


The dissipation of the plastic energy at a hinge line $L_{i}$ becomes (Jones [1], Sawczuk and Sokol-Supel [5])

$$
\dot{D}_{i}=\sum_{j=0}^{n} \int_{r_{j}}^{r_{j+1}} M_{j} \Delta \varphi_{i} r d r
$$

where the slope discontinuity at the hinge line at $\theta=\theta_{i}$

$$
\Delta \varphi_{i}=\left.\frac{\partial \dot{W}}{\partial n}\right|_{\theta_{i+}}-\left.\frac{\partial \dot{W}}{\partial n}\right|_{\theta_{i-}}
$$

Here $n$ stands for the normal direction to the line $L_{i}$.

However, if a continuous field of yield lines occurs then

$$
\dot{D}_{i}=\dot{W}_{0} \sum_{j=0}^{n} M_{j} \int_{r_{j}}^{r_{j+1}} \int_{0}^{2 \pi} \frac{1}{r_{*}}\left(1+2 \frac{r_{*}^{\prime 2}}{r_{*}^{2}}-\frac{r_{*}^{\prime \prime}}{r_{*}}\right) r d r d \theta+\dot{D}_{i c} .
$$

In eqn (13) the term $\dot{D}_{i c}$ corresponds to the dissipation of energy at the hinge formed at the clamped edge. Since the deflection rates correspond to (4), (5) one can conclude that

$$
\dot{D}_{i c}=\dot{W}_{0}(t) \Phi(\theta)
$$

where the second multiplier depends on the coordinate $\theta$ only.

Starting from the equality $\dot{D}_{i}=\dot{D}_{e}$ (see Jones [1]) and making use of eqns (7)-(14) one obtains

$$
\begin{gathered}
P \dot{W}_{0}-\mu \ddot{W}_{0} \dot{W}_{0} \int_{0}^{2 \pi} \sum_{j=0}^{n} \int_{r_{j}}^{r_{j+1}} h_{j}\left(1-\frac{r}{r_{*}}\right)^{2} r d r d \theta= \\
=\dot{W}_{0} \sum_{j=0}^{n} M_{j} \int_{r_{j}}^{r_{j+1}} \int_{0}^{2 \pi} \frac{1}{r_{*}}\left(1+2 \frac{r_{*}^{\prime 2}}{r_{*}^{2}}-\frac{r_{*}^{\prime \prime}}{r_{*}}\right) r d r d \theta+\dot{W}_{0} \Phi .
\end{gathered}
$$

It immediately follows from (15) that the acceleration

$$
\ddot{W}_{0}=\frac{1}{K}\left\{P-\Phi-\sum_{j=0}^{n} M_{j} \int_{r_{j}}^{r_{j+1}} \int_{0}^{2 \pi} \frac{1}{r_{*}}\left(1+\frac{r_{*}^{\prime 2}}{r_{*}^{2}}\right) r d r d \theta\right\}
$$


where

$$
K=\mu \int_{0}^{2 \pi} \sum_{j=0}^{n} \int_{r_{j}}^{r_{j+1}} h_{j}\left(1-\frac{r}{r_{*}}\right)^{2} r d r d \theta
$$

\section{Asymmetrically loaded circular plate}

Let us consider now an eccentrically loaded circular plate with stepped thickness. Let $R$ be the radius of the plate and $a$ - the distance between the center $O$ of the plate and the origin of coordinates $O_{1}$ (fig. 3). Assuming that the origin of coordinates is located at $O_{1}$ one can easily define.

$$
r_{n+1}=a \cos \theta+\sqrt{R^{2}-a^{2} \sin ^{2} \theta}
$$

In order to define the function $\Phi$ in (14) let us consider the hinge circle at the clamped edge of the plate. Let $A$ be the current point at the edge and $\alpha$ be the angle between $O A$ and the x-axis (fig. 3). Let $\psi$ be the angle between $O A$ and $O_{1} A$. In this case

$$
\frac{\partial \dot{W}}{\partial n}=\frac{\partial \dot{W}}{\partial x} \cos \alpha+\frac{\partial \dot{W}}{\partial y} \sin \alpha
$$

whereas

$$
\begin{aligned}
& \frac{\partial \dot{W}}{\partial x}=\frac{\partial \dot{W}}{\partial r} \cos \theta \\
& \frac{\partial \dot{W}}{\partial y}=\frac{\partial \dot{W}}{\partial r} \sin \theta
\end{aligned}
$$

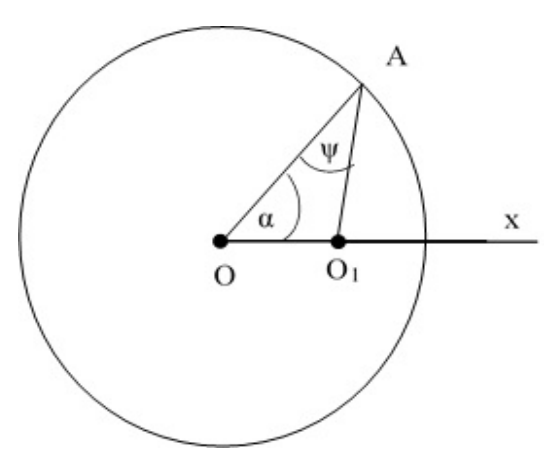

Figure 3: Hinge circle. 
It follows from (19), (20) that

$$
\frac{\partial \dot{W}}{\partial n}=-\frac{\dot{W}_{0}}{r_{*}} \cos \psi .
$$

Looking at the triangle $O O_{1} A$ in Fig. 3 one can easily conclude that

$$
\cos \psi=-\frac{1}{2 R r_{*}}\left(R^{2}+r_{*}^{2}-a^{2}\right) .
$$

Combining (21), (22) with (11)-(14) one can see that

$$
\Phi(\theta)=-M_{n} \frac{1}{2 R r_{*}^{2}}\left(R^{2}+r_{*}^{2}-a^{2}\right)
$$

where $r_{*}$ is defined by (18). Thus

$$
r_{*}^{\prime}=-a \sin \theta-\frac{a^{2} \sin \theta \cos \theta}{\sqrt{R^{2}-a^{2} \sin ^{2} \theta}} .
$$

where prim denotes the differentiation with respect to the angle $\theta$.

The accelerations can be calculated according to (16), (17) and (18), (24) separately for the stages when $P>0$ and when $P=0$, respectively.

During the first stage for $t \in\left(0, t_{1}\right)$

$$
\begin{gathered}
\dot{W}_{0}(t)=\ddot{W}_{0} t, \\
W_{0}(t)=\frac{1}{2} \ddot{W}_{0} t^{2} .
\end{gathered}
$$

Similarly for the final stage of motion for $t \in\left(t_{1}, t_{2}\right)$ one has

$$
\begin{gathered}
\dot{W}_{0}(t)=\ddot{W}_{1}\left(t-t_{1}\right)+\dot{W}_{0}\left(t_{1}\right), \\
W_{0}(t)=\frac{1}{2} \ddot{W}_{0}\left(t-t_{1}\right)^{2}+\dot{W}_{0}\left(t_{1}\right)\left(t-t_{1}\right)+W_{0}\left(t_{1}\right) .
\end{gathered}
$$

where $\ddot{W}_{1}$ denotes the acceleration at $r=0$ during the second stage of motion.

\section{Numerical results and discussion}

The plate with two different thicknesses $h_{0}$ and $h_{1}$ is studied in a greater detail. The circular plate loaded at the origin of coordinates is studied whereas

$$
h= \begin{cases}h_{0}, & r \in\left(0, r_{1}\right), \\ h_{1}, & r \in\left(r_{1}, r_{*}\right) .\end{cases}
$$

Let us denote $p=P / P_{S}$, where $P_{s}=2 \pi M_{0}$ is the static limit load, provided the load is applied at the center of the plate. The results of calculations are presented in Figs 4-6 where the maximal residual deflection versus the time 


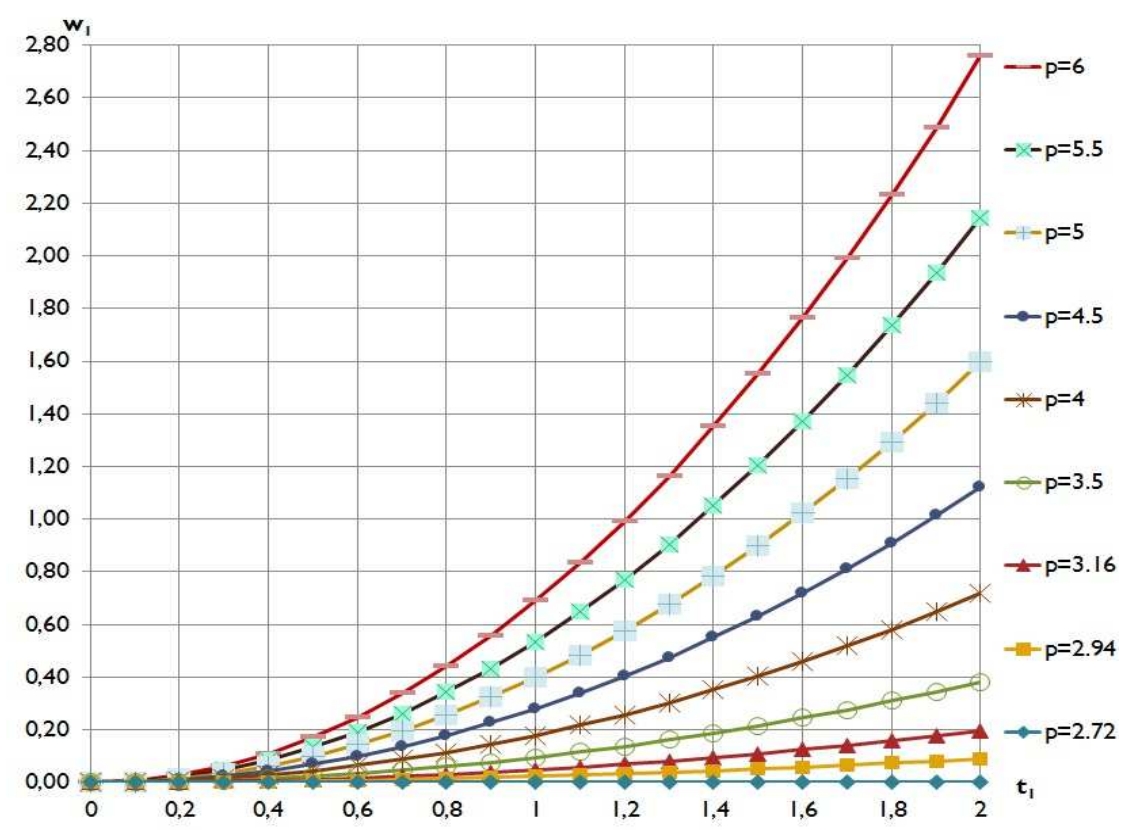

Figure 4: Maximal residual deflections $\left(r_{1}=0.2 R\right)$.

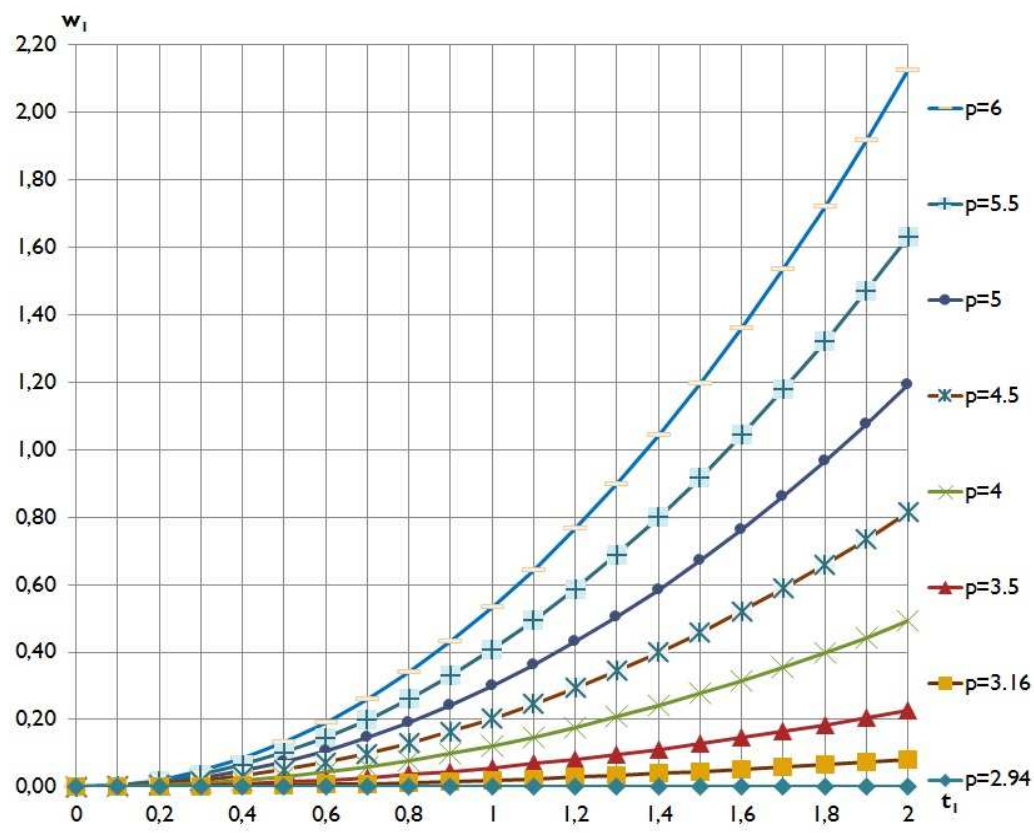

Figure 5: Maximal residual deflections $\left(r_{1}=0.5 R\right)$. 


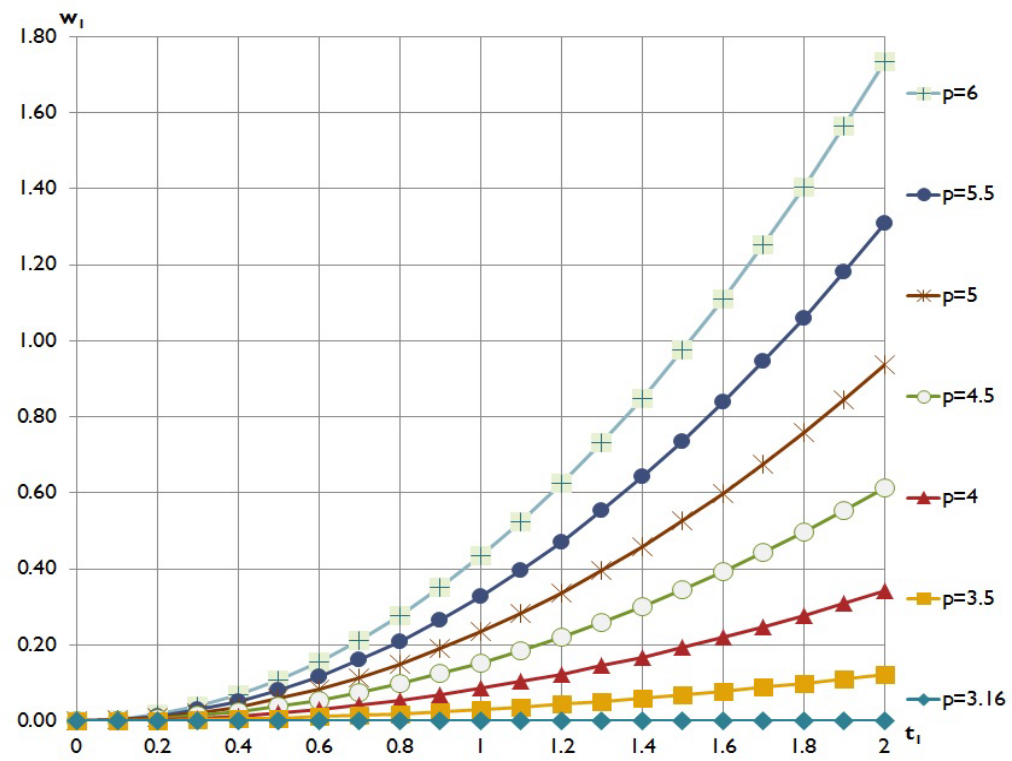

Figure 6: Maximal residual deflections $\left(r_{1}=0.8 R\right)$.

interval when the load is active is depicted. Here $a=0.1 R, h_{1}=0.8 h_{0}$. In fig. 4 $r_{1}=0.2 R$, in fig. $5 r_{1}=0.5 R$ and in fig. $6 r_{1}=0.8 R$. It can be seen from figs $4-$ 6 that the greater is $t_{1}$ the larger is the deflection $W_{1}$, as might be expected. On the other hand, the greater is the radius $r_{1}$ the smaller is corresponding residual deflection.

\section{Concluding remarks}

An approximate analysis of plates under impact loading was developed. The method is based on the classical method of "mode form motions". In the present paper this concept was extended to the case of non-axisymmetric bending of plates. Evidently, the method can be extended to the case of a distributed loading.

The method suggested above can accommodate plates made of functionally graded materials choosing the step locations and different thicknesses in accordance to the geometrical parameters at mesh points.

\section{Acknowledgements}

The support from the Estonian science Foundation through the Grant ETF9110 and from the Target financed project IUT 2057 are acknowledged. 


\section{References}

[1] Jones, N. Structural Impact. CUP, Cambridge, 575, 1989.

[2] Kaliszky, S. Dynamic plastic response of structures. In Sawczuk A, Bianchi G, editors, Plasticity Today: Modelling, Methods and Applications. Elsevier Science Publishers, London, pp. 787-820, 1984.

[3] Kaliszky, S. Plasticity. Theory and Engineering Applications. Elsevier, Amsterdam, 505 p, 1989.

[4] Lellep, J., Mürk, A. Asymmetric dynamic plastic behaviour of circular plates. Proceeding 2nd International Conference Optimization and Analysis of Structures. Tartu, pp. 70-75, 2013.

[5] Sawczuk, A., Sokol-Supel, J., Limit Analysis of Plates. Polish Scientific Publishers, PWN, Warszawa, 252 p, 1993.

[6] Symonds, P. S. Elastic, finite deflection and rate effects in a mode approximation technique for plastic deformation of pulse loaded structures, Journal of Mechanical Engineering Science, Vol. 22, pp. 189-197, 1980. 